\title{
Evidence of early disk-locking among low-mass members of the Orion Nebula Cluster (Corrigendum)
}

\author{
K. Biazzo ${ }^{1,2,3}$, C. H. F. Melo ${ }^{2}$, L. Pasquini' ${ }^{2}$, S. Randich ${ }^{1}$, J. Bouvier ${ }^{4}$, and X. Delfosse ${ }^{4}$ \\ 1 INAF - Osservatorio Astrofisico di Arcetri, Largo E. Fermi 5, 50125 Firenze, Italy \\ e-mail: kbiazzo@arcetri.astro.it \\ 2 ESO - European Southern Observatory, Karl-Schwarzschild-Str. 3, 85748 Garching bei München, Germany \\ 3 INAF - Osservatorio Astrofisico di Catania, via S. Sofia 78, 95123 Catania, Italy \\ ${ }^{4}$ Laboratoire d'Astrophysique, Observatoire de Grenoble, BP 53, 38041 Grenoble Cedex 9, France
}

A\&A, 508, 1301-1312 (2009), DOI: 10.1051/0004-6361/200913125

Key words. open clusters and associations: individual: Orion Nebula Cluster - stars: low-mass - stars: pre-main sequence stars: late-type - accretion, accretion disks - errata, addenda

An error occurred during the production process. Figure 8 was published twice. The corrected Figs. 6 and 8 are published below.

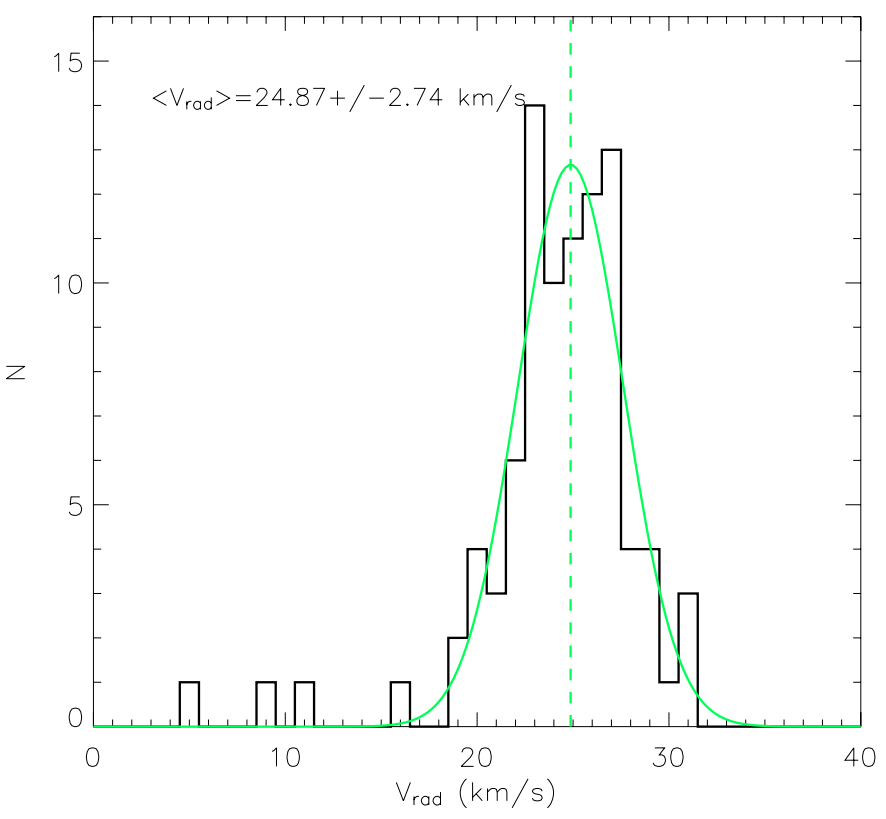

Fig. 6. Radial velocity distribution for our sample of stars with $M<$ $0.25 M_{\odot}$. For the most probable binaries we plot the mean values of the two components (Table 2). The Gaussian fit of the distribution yields a mean $V_{\mathrm{rad}}$ of $24.87 \mathrm{~km} \mathrm{~s}^{-1}$ (with a $\sigma_{V \mathrm{rad}}$ of $2.74 \mathrm{~km} \mathrm{~s}^{-1}$ ), which is fully consistent with the membership in the ONC (e.g., Stassun et al. 1999).

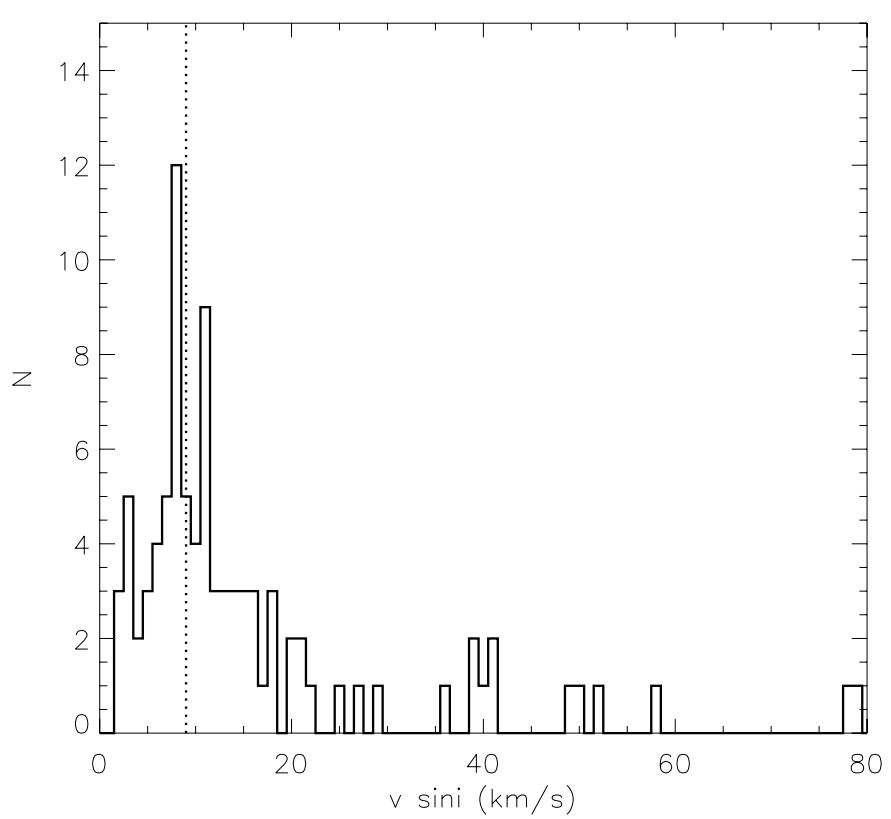

Fig. 8. $v \sin i$ distribution for our low-mass stars. Our $v \sin i$ limit of $9 \mathrm{~km} \mathrm{~s}^{-1}$ is shown with a dotted line.

\section{References}

Stassun, K. G., Mathieu, R. D., Mazeh, T., \& Vrba, F. J. 1999, AJ, 117, 2941 